\title{
Sustained postinfarction myocardial oedema in humans visualised by magnetic resonance imaging
}

\author{
J C Nilsson, G Nielsen, B A Groenning, T Fritz-Hansen, L Sondergaard, G B Jensen, \\ H B W Larsson
}

\begin{abstract}
Objective-To demonstrate postinfarction myocardial oedema in humans with particular reference to the longitudinal course, using magnetic resonance imaging (MRI).

Design-Prospective observational study. Subjects were studied one week, one month, three months, six months, and one year after presenting with a myocardial infarct.

Setting-Cardiology and magnetic resonance departments in a Danish university hospital.

Patients-10 patients (three women, seven men), mean (SEM) age 58.2 (3.20) years, with a first transmural myocardial infarct.

Main outcome measures-Location and duration of postinfarction myocardial oedema.

Results-All patients had signs of postinfarction myocardial oedema. The magnetic resonance images were evaluated by two blinded procedures, employing two MRI and two ECG observers: (1) MRI determined oedema location was compared with the ECG determined site of infarction and almost complete agreement was found; (2) the time course of postinfarction myocardial oedema was explored semiquantitatively, using an image ranking procedure. Myocardial oedema was greatest at the initial examination one week after the infarction, with a gradual decline during the following months (Spearman's rank correlation analysis: $\rho_{\text {observer } 1}=0.94(p<0.0001)$ and $\left.\rho_{\text {observer } 2}=0.97(\mathrm{p}<0.0001)\right)$. The median duration of oedema was six months.

Conclusions-Postinfarction myocardial oedema seems surprisingly long lasting. This observation is of potential clinical interest because the oedema may have prognostic significance.

(Heart 2001;85:639-642)
\end{abstract}

Keywords: myocardial infarction; myocardial oedema; magnetic resonance imaging

Myocardial oedema induced by ischaemia and enhanced by subsequent reperfusion has been known for years. The pathogenesis is complex and not completely understood, but fundamentally implicates accumulation of osmotically active substances within the ischaemic region which causes an influx of water. ${ }^{1-3}$ As this accumulation takes place in the intracellular as well as the extracellular spaces, so also does the ensuing water influx. The final result is combined cellular and interstitial oedema. In addition to osmotic forces, a thermodynamic dysequilibrium contributes to the intracellular flux of water. ${ }^{45}$ Water enters the ischaemic region mainly through collateral vessels and to a lesser extent from the surrounding tissue, and through retrograde flow in venous and lymphatic vessels. ${ }^{5}$ If reperfusion subsequently takes place, the flow of normo-osmotic blood into this hyperosmotic region further enhances the oedema.

In vivo visualisation of myocardial oedema has been difficult until the recent development of magnetic resonance imaging (MRI) pulse sequences, which combine a particular sensitivity to oedema with the ability to eliminate flow and motion artefacts. ${ }^{6}$ Our aim in the present study was to demonstrate postinfarction myocardial oedema in humans using this technique, with particular reference to the longitudinal course of the oedema.

\section{Methods}

PATIENTS

The study was approved by the regional ethics committee, and all the participating subjects supplied written informed consent. Ten patients with their first transmural myocardial infarct were studied (table 1). Creatine kinase $\mathrm{MB}$ (CK-MB) was measured on admission and after 4, 8, 12, 24, and 48 hours. ECGs were obtained on admission, at one hour after thrombolytic treatment, and then once daily. None of the patients suffered from additional ischaemic events during the investigation.

MAGNETIC RESONANCE IMAGING

Each patient was examined up to five times. The first examination was performed between seven and nine days after the myocardial infarct $(n=10)$, followed by examinations after one month ( $\mathrm{n}=9)$, three months $(\mathrm{n}=8)$, six months $(n=9)$, and one year $(n=9)$. MRI was conducted using a 1.0 Tesla whole body scanner (Impact Expert Magnetom, Siemens AG, Erlangen, Germany) equipped with a standard phased array chest coil. In order to visualise myocardial oedema, the entire left ventricle was sliced in the true short axis from apex to basis, using a T2 weighted shortinversion-time, inversion-recovery (STIR) breath hold pulse sequence ${ }^{6}$ (parameter settings: repetition time $(\mathrm{TR})=2 \times \mathrm{RR}$ interval; echo time $(\mathrm{TE})=64 \mathrm{~ms}$; field of view $(\mathrm{FOV})=$ $263 \times 350 \mathrm{~mm}^{2}$; matrix $=138 \times 256$; slice thickness $=10 \mathrm{~mm}$ ). Typically, the left ventricle was covered by 9-11 slices. Each STIR image (slice) was obtained at end diastole by ECG triggered segmented data acquisition over 14 heart cycles. Imaging was performed without the use of contrast agents. 
Table 1 Patient characteristics and results

\begin{tabular}{|c|c|c|c|c|c|c|c|c|}
\hline Patient & Sex & Age (years) & $\begin{array}{l}C K-M B \max \\
(\mu g / l)\end{array}$ & $\begin{array}{l}\text { Delay in } \\
\text { thrombolysis (hours) }\end{array}$ & Reperfusion & $\begin{array}{l}\text { Infarct location by } \\
E C G\end{array}$ & $\begin{array}{l}\text { Oedema location } \\
\text { by MRI }\end{array}$ & $\begin{array}{l}\text { Duration of } \\
\text { oedema (months) }\end{array}$ \\
\hline 1 & M & 51 & 271 & - & Yes & Ant-sep & Ant-sep & 6 \\
\hline 2 & $M$ & 75 & 331 & 4.0 & Yes & Ant-sep-lat & Ant-sep & 1 \\
\hline 3 & M & 53 & 213 & 3.0 & Yes & Inf & Inf & 1 week \\
\hline 4 & M & 74 & 383 & 3.0 & Yes & Lat & Lat & 3 \\
\hline 5 & M & 51 & 423 & 2.0 & Yes & Ant-sep & Ant-sep & 12 \\
\hline 6 & $\mathrm{~F}$ & 65 & 136 & 5.0 & No & Inf & Inf & 1 \\
\hline 7 & M & 55 & 152 & 6.0 & Yes & Ant-sep-lat & Lat & 12 \\
\hline 8 & M & 60 & 225 & 5.0 & No & Ant-sep & Ant-sep & 6 \\
\hline 9 & $\mathrm{~F}$ & 45 & 136 & 1.5 & Yes & Ant-sep & Ant-sep & 6 \\
\hline 10 & $\mathrm{~F}$ & 53 & 607 & 4.0 & Yes & Ant-sep & Ant-sep & 12 \\
\hline
\end{tabular}

Ant, anterior; CK-MBmax, maximum value of creatine kinase MB isoenzyme; Duration of oedema, the latest MRI examination showing high signal intensity areas within the myocardium; Inf, inferior; Infarct location, defined by ECGs obtained on admission; Lat, lateral; MRI, magnetic resonance imaging; Oedema location, defined by the initial MRI examination; Reperfusion, defined by $\mathrm{ECG}^{7}$; Sep, septal; Delay in thrombolysis, time from appearance of symptoms to start of thrombolytic treatment.

\section{Results}

In STIR images, oedematous myocardium is depicted as high signal intensity areas (fig 1). For subsequent evaluation, all images were transferred to glossy paper using a high quality printing technique. All examinations in each patient were printed with identical contrast and brightness by adjusting the centre and window settings until the visual appearance of the liver was the same.

The STIR images were evaluated by two blinded procedures employing two MRI observers and two ECG observers.

MRI determined oedema location was compared with the ECG determined site of infarction. Oedema location and the site of infarction were defined as being anterior, septal, inferior, or lateral, including combinations. Almost complete agreement was found between the
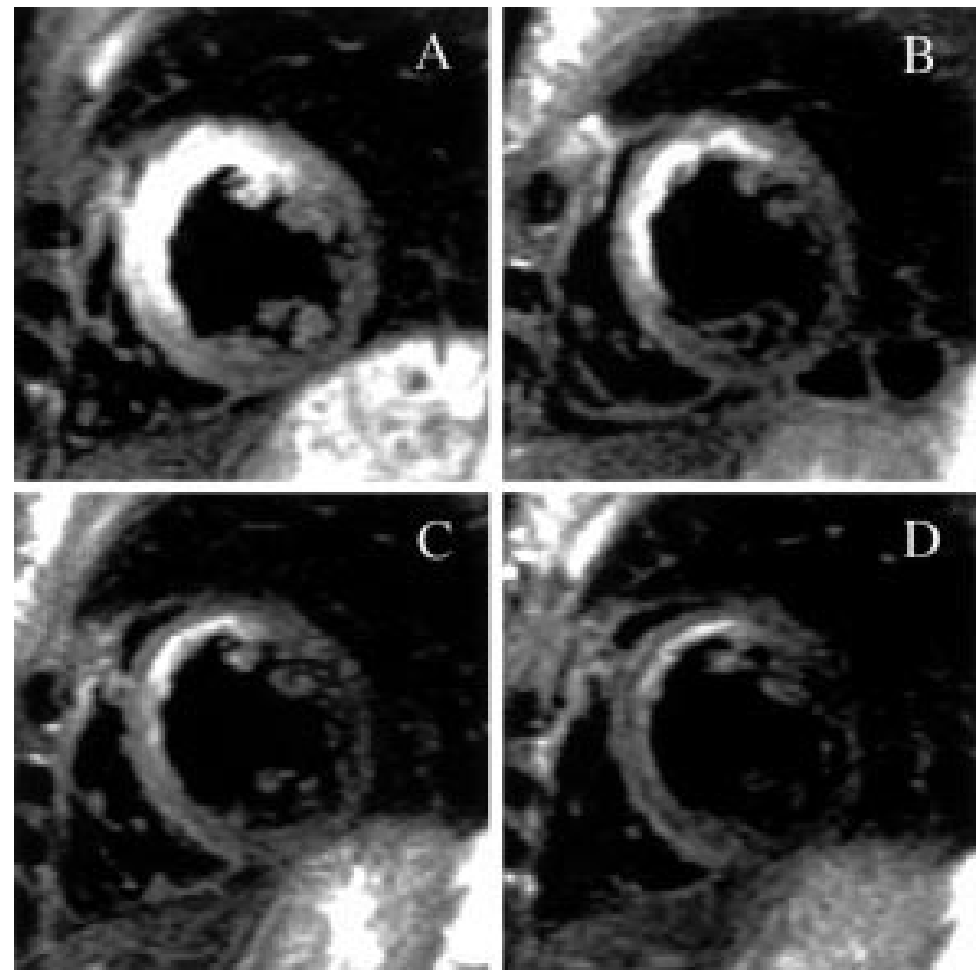

Figure 1 The course of postinfarction myocardial oedema (table 1, patient 9). Corresponding slices of the right and left ventricles positioned in the true short axis at mid-ventricular level from four succeeding examinations. (A) Seventh day post-myocardial infarction; (B) one month; (C) three months; (D) six months. The high signal intensity (bright) areas within the myocardium represent oedema. Notice the large extent of oedema on the seventh day affecting particularly the septum and anterior wall of the left ventricle, and the gradual decline of the oedema over the following months.
MRI and ECG observers' indication of the expected location of oedema in the infarct region (table 1 ).

In order to explore the time course of postinfarction myocardial oedema in a semiquantitative manner, the MRI observers performed ranking of all examinations in each patient. Ranking was done according to decreasing extent and brightness of the myocardial high signal intensity areas. Analysis of these data revealed that the oedematous areas were of greatest extent at the initial examination after approximately one week, with a gradual decline during the ensuing months. The decline over time was significant (Spearman's rank correlation analysis: $\rho_{\text {observer } 1}=0.94$ $(\mathrm{p}<0.0001)$ and $\left.\rho_{\text {observer } 2}=0.97(\mathrm{p}<0.0001)\right)$. The interobserver agreement was good (weighted $\kappa$ analysis: $\kappa_{\mathrm{w}}=0.91(95 \%$ confidence interval (CI) 0.84 to 0.99 )).

The minimum duration of oedema in a particular area varied among patients from one week to 12 months, with a median of six months (table 1). In case of interobserver disagreement, the estimation of shortest oedema duration was chosen. There was complete agreement between the two observers in seven patients. In two patients, the observers disagreed over one examination, and in one patient they disagreed over two examinations. The interobserver agreement was good (weighted $\kappa$ analysis: $\kappa_{\mathrm{w}}=0.73(95 \%$ CI 0.44 to 1.00$)$ ).

We found no relation between the duration of oedema and delay in thrombolysis, reperfusion status, or CK-MBmax.

\section{Discussion}

Our study shows the presence of prolonged postinfarction myocardial oedema in humans. All 10 patients displayed signs of myocardial oedema, with its localisation corresponding to the ECG determined site of infarction.

DURATION OF POSTINFARCTION MYOCARDIAL OEDEMA

The oedema was of greatest extent at the initial examination, approximately one week after the myocardial infarct, and it declined gradually afterwards, probably over a period of several months. Interobserver agreement was very good. No data were obtained during the first week, but previous studies suggest that the oedema might accumulate within hours of the ischaemic insult if reperfusion takes place, ${ }^{358}$ 
and otherwise within days. ${ }^{8}$ With regard to the duration of postinfarction myocardial oedema, Wisenberg and colleagues studied dogs subjected to either permanent ischaemia or ischaemia followed by reperfusion. ${ }^{8}$ At their final examination after three weeks, myocardial water content, as measured by histopathological methods, was still significantly increased in the infarct zone in both groups. In the present study, persistence of the myocardial high signal intensity areas varied from one week to 12 months, with a median of six months.

At the initial examinations, the high signal intensity areas almost certainly represent oedema. ${ }^{6-10}$ In the later examinations, other conditions such as deposition of haemoglobin derivatives or increased ratio of unbound to bound water in the absence of oedema might be hypothetical explanations. However, it is unlikely for two reasons that the high signal intensity areas signify deposition of haemoglobin derivatives. First, the appearance of the areas was homogeneous, in contrast to the well described heterogeneous or "patchy" appearance of experimentally induced myocardial haemorrhages, ${ }^{11}$ as well as haemorrhages in other organs such as the brain. ${ }^{12}$ Second, the only known haemoglobin derivative to cause signal enhancement with the technique applied is methaemoglobin, and it is unlikely that this substance was present in the insult region after several months. ${ }^{12}$ Prolonged presence of oedema may be explained by compromised drainage of the infarct region owing to vessel damage and reparative processes generating scar tissue. ${ }^{5}$ As these reparative processes last for months, ${ }^{13}$ it is likely that the high signal intensity areas in the MRI examinations performed several months after the ischaemic insult represent persisting oedema, although a condition characterised by an increased fraction of unbound water without oedema cannot definitely be ruled out.

POSTINFARCTION MYOCARDIAL OEDEMA AND OTHER MRI TECHNIQUES

Visualisation of myocardial infarct by gadolinium enhanced $\mathrm{MRI}^{14-22}$ as well as by sodium $\mathrm{MRI}^{23-26}$ is subject to ongoing debate. In a recent study ${ }^{20}$ using a refined MRI technique, the transmural extent of irreversibly injured myocardium was accurately delineated by delayed gadolinium enhancement. In addition, regions of delayed gadolinium enhancement in MRI studies performed eight weeks after the ischaemic insult represented chronic infarction. The mechanisms responsible for the gadolinium enhancement in myocardial infarction are incompletely understood, but may involve an increased volume of distribution of the contrast agent in the insult region. Gadolinium chelates are distributed primarily in the extracellular compartment, so such an increase could be explained by interstitial oedema and particularly by cellular oedema with subsequent disintegration of the plasma membrane. ${ }^{18} 19$ Thus gadolinium enhancement following myocardial infarction may in part reflect myocardial oedema.
Recent studies have also shown that myocardial high signal intensity regions in sodium MRI represent areas of irreversible ischaemic injury. ${ }^{2526}$ The basis for infarct visualisation by sodium MRI is an increase in sodium concentration in necrotic regions, which also appears to be related to myocardial oedema. Initially, cell membrane dysfunction caused by ischaemia seems to result in intracellular sodium accumulation with subsequent cellular oedema. ${ }^{4}$ Rupture of myocytes in combination with minor interstitial oedema may follow, causing an overall increase in sodium concentration in the infarct region, because extracellular fluid is rich in sodium. ${ }^{25} 26$

Thus visualisation of myocardial infarction by sodium or gadolinium enhanced MRI and visualisation of postinfarction myocardial oedema by MRI using the STIR technique may be different ways of looking at the same phenomenon. In consequence, the experimental results of Kim and colleagues showing delayed myocardial contrast enhancement eight weeks after experimentally induced myocardial infarction may support our observations of sustained postinfarction oedema. ${ }^{20}$

CLINICAL SIGNIFICANCE

Our study failed to show any relation between the duration of oedema and thrombolytic delay, reperfusion status, or infarct size as estimated by CK-MBmax. This may in part reflect the small number of observations, and also the somewhat imprecise measure of reperfusion status applied in the study.

The clinical significance of postinfarction myocardial oedema has not been established so far. However, experimental results indicate that the oedema might adversely affect ventricular contraction and relaxation, ${ }^{27-31}$ initiate arrhythmia ${ }^{32}$ and induce additional necrosis. ${ }^{12}$ Consequently, detection and quantification of myocardial oedema is of potential clinical interest. Visualisation by MRI is attractive, as the method is non-invasive and without any known risks, thus allowing repeated examinations. Additionally, the STIR technique integrates perfectly with other MRI techniques which provide detailed structural and haemodynamic information on the postinfarction heart. In future MRI studies, it would obviously be interesting to study the exact relation between myocardial oedema and different patterns of gadolinium enhancement in addition to short and long term ventricular systolic and diastolic function.

CONCLUSIONS

Prolonged postinfarction myocardial oedema was demonstrated in 10 patients by MRI. Myocardial oedema was of greatest extent at the initial examination one week after the infarction and declined thereafter, probably over a period of several months. As myocardial oedema may have consequences for prognosis, in vivo detection is of potential clinical interest.

We gratefully acknowledge the supporting grants from the Danish Heart Foundation (No 97-1-3-62-22491, 97-2-4-56-22548, 98-1-4-55-22598, and 99-1-3-62-22698). 
1 Tranum JJ, Janse MJ, Fiolet WT, et al. Tissue osmolality, cell swelling, and reperfusion in acute regional myocardial ischemia in the isolated porcine heart. Circ Res 1981;49:364-81.

2 Steenbergen C, Hill ML, Jennings RB. Volume regulation and plasma membrane injury in aerobic, anaerobic, and ischemic myocardium in vitro. Effects of osmotic cell swelling on plasma membrane integrity. Circ Res 1985;57:86475 .

3 Jennings RB, Schaper J, Hill ML, et al. Effect of reperfusion late in the phase of reversible ischemic injury. Changes in cell volume, electrolytes, metabolites, and ultrastructure. Circ Res 1985;56:262-78.

4 Tomita M, Gotoh F. Cascade of cell swelling: thermodynamic potential discharge of brain cells after membrane injury. Am f Physiol 1992;262:H603-10.

5 Garcia DD, Oliveras J. Myocardial oedema: a preventable cause of reperfusion injury? Cardiovasc Res 1993;27:155563.

6 Simonetti OP, Finn JP, White RD, et al. "Black blood" T2-weighted inversion-recovery MR imaging of the heart. T2-weighted inversion-recove
Radiology 1996;199:49-57.

7 Clemmensen P, Ohman EM, Sevilla DC, et al. Changes in standard electrocardiographic ST-segment elevation predictive of successful reperfusion in acute myocardial infarction. Am f Cardiol 1990;66:1407-11.

8 Wisenberg G, Prato FS, Carroll SE, et al. Serial nuclear magnetic resonance imaging of acute myocardial infarction with and without reperfusion. Am Heart f 1988;115:51018.

9 Garcia DD, Oliveras J, Gili J, et al. Analysis of myocardial oedema by magnetic resonance imaging early after coronary artery occlusion with or without reperfusion [published erratum appears in Cardiovasc Res 1993;27: 1889]. Cardiovasc Res 1993;27:1462-9.

10 Boxt LM, Hsu D, Katz J, et al. Estimation of myocardial water content using transverse relaxation time from dual spin-echo magnetic resonance imaging. Magn Reson Imaging 1993;11:375-83.

11 Lotan CS, Bouchard A, Cranney GB, et al. Assessment of postreperfusion myocardial hemorrhage using proton postreperfusion myocardial hemorrhage using proto
NMR imaging at $1.5 \mathrm{~T}$. Circulation $1992 ; 86: 1018-25$.

12 Mattle HP, Edelman RR, Schroth G, et al. Spontaneous and traumatic hemorrhage. In: Edelman RR, Zlatkin MB, Hesselink JR, eds. Clinical magnetic resonance imaging. Philadelphia: WB Saunders Co, 1996:652-702.

13 Hackel DB, Jennings RB. The heart. In: Rubin E, Farber JL eds. Pathology. Philadelphia: JB Lippincott Co, 1988:496541 .

14 Schaefer S, Malloy CR, Katz J, et al. Gadolinium-DTPAenhanced nuclear magnetic resonance imaging of reperfused myocardium: identification of the myocardial bed at risk. F Am Coll Cardiol 1988:12:1064-72.

15 de-Roos A, van-Rossum AC, van-der-Wall E, et al. Reperfused and nonreperfused myocardial infarction: diagnostic potential of Gd-DTPA-enhanced MR imaging. Radiology 1989;172:717-20.

16 van-der-Wall $\mathrm{EE}$, van-Dijkman $\mathrm{PR}$, de-Roos $\mathrm{A}$, et al. Diagnostic significance of gadolinium-DTPA (diethylenetriamine penta-acetic acid) enhanced magnetic resonance imaging in thrombolytic treatment for acute myocardial infarction: its potential in assessing reperfusion. Br Heart $\mathcal{F}$ 1990;63:12-17.

17 Holman ER, van-Jonbergen HP, van-Dijkman PR, et al. Comparison of magnetic resonance imaging studies with enzymatic indexes of myocardial necrosis for quantification of myocardial infarct size. Am $\mathcal{F}$ Cardiol 1993;71:1036-40.

18 Lima JA, Judd RM, Bazille A, et al. Regional heterogeneity of human myocardial infarcts demonstrated by contrastenhanced MRI Potential mechanisms. Circulation 1995;92: $1117-25$.

19 Judd RM, Lugo OC, Arai M, et al. Physiological basis of myocardial contrast enhancement in fast magnetic resonance images of 2-day-old reperfused canine infarcts. Circulation 1995;92:1902-10

20 Kim RJ, Fieno DS, Parrish TB, et al. Relationship of MRI delayed contrast enhancement to irreversible injury, infarct age, and contractile function. Circulation 1999;100:19922002.

21 Hillenbrand HB, Kim RJ, Parker MA, et al. Early assessment of myocardial salvage by contrast-enhanced magnetic resonance imaging. Circulation 2000;102:1678-83.

22 Kim RJ, Wu E, Rafael A, et al. The use of contrast-enhanced magnetic resonance imaging to identify reversible myocar-
dial dysfunction. N Engl $\mathcal{F}$ Med 2000;343:1445-53.

23 Cannon PJ, Maudsley AA, Hilal SK, et al. Sodium nuclear magnetic resonance imaging of myocardial tissue of dogs after coronary artery occlusion and reperfusion. $\mathcal{F} \mathrm{Am} \mathrm{Coll}$ Cardiol 1986;7:573-9.

24 Parrish TB, Fieno DS, Fitzgerald SW, et al. Theoretical basis for sodium and potassium MRI of the human heart at 1.5 T. Magn Reson Med 1997;38:653-61.

25 Kim RJ, Judd RM, Chen EL, et al. Relationship of elevated ${ }^{23} \mathrm{Na}$ magnetic resonance image intensity to infarct size after acute reperfused myocardial infarction. Circulation 1999;100:185-92.

26 Rochitte CE, Kim RJ, Hillenbrand HB, et al. Microvascular integrity and the time course of myocardial sodium accumulation after acute infarction. Circ Res 2000;87:64855.

27 Laine GA. Change in (dP/dt)max as an index of myocardial microvascular permeability. Circ Res 1987;61:203-8.

28 Davis KL, Mehlhorn U, Laine GA, et al. Myocardial edema, left ventricular function, and pulmonary hypertension. $\mathcal{F}$ Appl Physiol 1995;78:132-7.

29 Rubboli A, Sobotka PA, Euler DE. Effect of acute edema on left ventricular function and coronary vascular resistance in the isolated rat heart. Am f Physiol 1994;267:H1054-61.

30 Pratt JW, Schertel ER, Schaefer SL, et al. Acute transient coronary sinus hypertension impairs left ventricular function and induces myocardial edema. Am 7 Physiol 1996;271:H834-41

31 Miyamoto M, McClure DE, Schertel ER, et al. Effects of hypoproteinemia-induced myocardial edema on left ventricular function. Am $\mathcal{F}$ Physiol 1998;274:H937-44.

32 Sorota S. Swelling-induced chloride-sensitive current in canine atrial cells revealed by whole-cell patch-clamp method. Circ Res 1992;70:679-87. 